\title{
BMJ Open Primary healthcare system readiness to prevent and manage non-communicable diseases in Bangladesh: a mixed-method study protocol
}

\author{
Ashraful Kabir (D) , Md Nazmul Karim, Baki Billah
}

To cite: Kabir A, Karim MN, Billah B. Primary healthcare system readiness to prevent and manage noncommunicable diseases in Bangladesh: a mixed-method study protocol. BMJ Open 2021;11:e051961. doi:10.1136/ bmjopen-2021-051961

- Prepublication history for this paper is available online. To view these files, please visit the journal online (http://dx.doi. org/10.1136/bmjopen-2021051961).

Received 02 April 2021 Accepted 23 August 2021

Check for updates

(c) Author(s) (or their employer(s)) 2021. Re-use permitted under CC BY-NC. No commercial re-use. See rights and permissions. Published by BMJ.

Department of Epidemiology and Preventive Medicine, School of Public Health and Preventive Medicine, Monash University, Melbourne, Victoria, Australia

Correspondence to

Ashraful Kabir;

md.kabir@monash.edu

\section{ABSTRACT}

Introduction The burden of non-communicable diseases (NCDs) is rapidly increasing in Bangladesh. Currently, it contributes to $67 \%$ of annual deaths, and accounts for approximately $64 \%$ of the disease burden. Since $70 \%$ of the Bangladeshi population residing in the rural area rely on the primary healthcare system, assessment of its capacity is crucial for guiding public health decisions to prevent and manage NCDs. This protocol is designed to recognise and assess the Bangladeshi health system's readiness for NCDs at the primary level.

Methods and analysis The study will use a mixedmethod design. Numerical data will be collected using households and health facilities surveys, while qualitative data will be collected by interviewing healthcare providers, policy planners, health administrators and community members. The WHO's Service Availability and Readiness Assessment (SARA) methodology and Package of Essential Non-communicable (PEN) Disease Interventions for Primary Healthcare reference manuals will be used to assess the readiness of the primary healthcare facilities for NCD services. Furthermore, Health System Dynamics Framework will be used to examine health system factors. Using the supportive items outlined in the WHO PEN package, and indicators proposed in WHO SARA methodology, a composite score will be created to analyse facility-level data. Two independent samples t-test, analysis of variance and $\chi^{2}$ test methods will be used for bivariate analysis, and multiple regression analysis will be used for multivariable analysis. Complementarily, the thematic analysis approach will be used to analyse qualitative data.

Ethics and dissemination The project has been approved by the Monash University Human Research Ethics Committee (Project ID: 27112), and Bangladesh Medical Research Council (Ref: BMRC/NREC/20192022/270). The research findings will be shared through research articles, conference proceedings or in other scientific media. The reports or publications will not have any information that can be used to identify any of the study participants.

\section{INTRODUCTION}

Non-communicable diseases (NCDs) have become the dominant cause of disabilities and deaths globally, resulting in the loss of
Strengths and limitations of this study

- Mixed-method design offers a deeper and comprehensive understanding of the readiness of the healthcare system to manage non-communicable diseases (NCDs) at the primary healthcare settings of a country.

- The proposed methods and conceptual framework will examine demand and supply-side enablers and barriers to the readiness of primary healthcare system, both essential considerations in addressing NCDs.

- The mixed-method design will gather data from the participants with various roles and backgrounds and regions of the country, which will increase the validity and trustworthiness of this study's findings.

- Considering high prevalence of the major NCDs (diabetes mellitus, chronic respiratory diseases, cardiovascular disease and cancer), the scope of service available at the primary healthcare level, and the current strategic and programmatic priority, the proposed study will include only the major NCDs, which means the findings of this study may not be generalisable to other NCDs defined locally in Bangladesh.

41 million lives in 2018, and accounting for $71 \%$ of all deaths, over three-quarters of which occurred in low-income and middle-income countries. ${ }^{12}$ It has been projected that NCDrelated deaths will reach 52 million by 2030 , if the current trends continue. ${ }^{3}$ A joint study conducted by the World Economic Forum and Harvard University showed that NCDrelated costs will increase to US\$47 trillion, equivalent to $75 \%$ of global domestic product from 2010 to 2030, if appropriate measures are not taken. ${ }^{4}$

Primary healthcare (PHC) has been recognised as a powerful strategy as well as a practical approach for preventing and managing NCDs because of its wide range of coverage, cost-effectiveness and healthcare infrastructure. ${ }^{5}$ As such, the WHO and the UNICEF emphasised, in a joint declaration, 
the importance and adoption of the PHC approach to achieve global health goals. ${ }^{6}$ In past decades, numerous efforts have also been proposed and/or advocated to prepare the PHC system as an effective mechanism for preventing and managing NCDs at the individual and population levels. ${ }^{7-10}$ Several studies in South Asia, ${ }^{11-14}$ Southeast Asia ${ }^{15-17}$ and sub-Sharan Africa ${ }^{18-20}$ investigated the PHC system readiness for NCDs from the supply-side perspective, mainly the facility-level readiness using the WHO Service Availability and Readiness Assessment (SARA) reference manual and/or WHO package of essential non-communicable (PEN) intervention package. However, the demand-side aspect of PHC system readiness, such as the community characteristics and associated determinants, remains largely underexplored.

In recent years, Bangladesh has made remarkable advances in health outcomes with declined maternal as well as child mortality, and fertility rates, increased longevity, coverage of vaccinations, and vitamin $\mathrm{A}$ and $\mathrm{D}$ supplementation. ${ }^{21-23}$ However, the World Bank reported that the country is currently undergoing sociodemographic and epidemiological transitions. ${ }^{24}$ Demographic transitions such as increasing longevity and decreasing fertility are thus fuelling shifts in disease epidemiology, wherein NCDs are becoming evident, and infectious diseases are taking a back seat. ${ }^{25}{ }^{26}$ Studies conducted in various geographical locations (rural/urban settings), among different age groups, sexes and ethnicities in Bangladesh showed that the prevalence of NCDs had been rapidly increasing over the last decades. ${ }^{27-30}$ The Global Burden of Diseases study reported that the proportion of deaths from NCDs gradually increased in Bangladesh from $43.4 \%$ in 2000 to $66.9 \%$ in $2015 .^{31}$ In 2016 , an estimated 856000 deaths (67\% of total deaths) were attributed to NCDs, which was approximately $64 \%$ of the country's disease burden. ${ }^{32}$ Previous studies also noted that an alarming increase of NCD-related factors such as life-style changes (sedentary behaviour), unhealthy diets (processed/highly flavoured foods) and harmful use of tobacco products ${ }^{28} 33$ will lead to the rise of NCDs in the coming years unless appropriate measures are taken. ${ }^{34-37}$

Historically, in Bangladesh, the PHC system focused on responding to acute conditions, primarily infectious diseases and parasitic infestations. ${ }^{25} 38$ A large share of healthcare resources and the PHC workforce being deployed mainly for family planning as well as maternal and child health services. ${ }^{25} 38$ Consequently, the focus on NCDs remained less prioritised over the decades. Studies showed that the shifting of epidemiological and demographic conditions could pose new challenges (ageing population, urban sprawl, environmental degradation) for Bangladesh's PHC system. ${ }^{24} 3940$ NCD-related services are required for continuous facility-based healthcare and family support, involving higher treatment costs, skilled and qualified healthcare workforces, constant supplies of medical equipment, medicines and so forth. Presumably, people living in rural settings (approximately $70 \%$ of the country's total population) ${ }^{41}$ are likely to be substantially affected by NCDs due to insufficient healthcare facilities and infrastructure, unavailability of healthcare professionals, lower socioeconomic conditions (eg, poverty and income inequality) and disadvantaged social positions (eg, illiteracy, lack of awareness and poor health education). ${ }^{42} 43$

The PHC system in Bangladesh has an extensive network of healthcare facilities at the upazila (subdistrict) level (figure 1), which is responsible for delivering basic healthcare services (vaccination, reproductive and child healthcare, nutrition and health education, screening of NCDs, treating common disease and referral to higher facilities) in both community and facility levels. ${ }^{44}$ Although some recent studies addressed the prevalence of NCDs and its associated factors, ${ }^{27-29} 394546$ the capacity of Bangladesh's PHC system, which is the first-line contact for the health needs of approximately two-thirds of the population, ${ }^{33} 45$ has not been adequately investigated. A few studies were conducted with a specific focus on service availability or general readiness of the facilities at the upazila health complex (UHC) (primary-level hospitals located at the subdistrict), and district hospitals (secondary-level hospitals located in the district) for diabetes and/or hypertension. ${ }^{12}{ }^{47}$ However, a comprehensive analysis of the readiness of the PHC system (ie, service, workforce, information systems, supplies and logistics, leadership and governance, and financing) largely remain under-researched. Given the importance of better planning, best use of resources and ensuring complete health needs, the readiness of the primarylevel healthcare system is vital to address the rising burden of NCDs. Thus, this study protocol is designed to evaluate research questions/objectives regarding the primary health system's readiness to prevent and manage NCDs. The outcomes of this study protocol will address a critical information gap, and eventually help to guide public health decisions for preventing and managing NCDs in Bangladesh and similar settings elsewhere.

\section{Research objective}

\section{General objective}

The overall objective of this study protocol is to outline a study design to explore the PHC system's readiness to prevent and manage NCDs, which is comprised of the following specific objectives.

\section{Specific objectives}

1. To assess the readiness of the Bangladesh PHC system in preventing and managing NCDs.

2. To evaluate the characteristics of community members and their contexts for receiving services from the PHC level for preventing and managing NCDs.

3. To identify gaps and opportunities in existing NCDs prevention and management services at the PHC system, and explore feasible ways for service optimisation. 


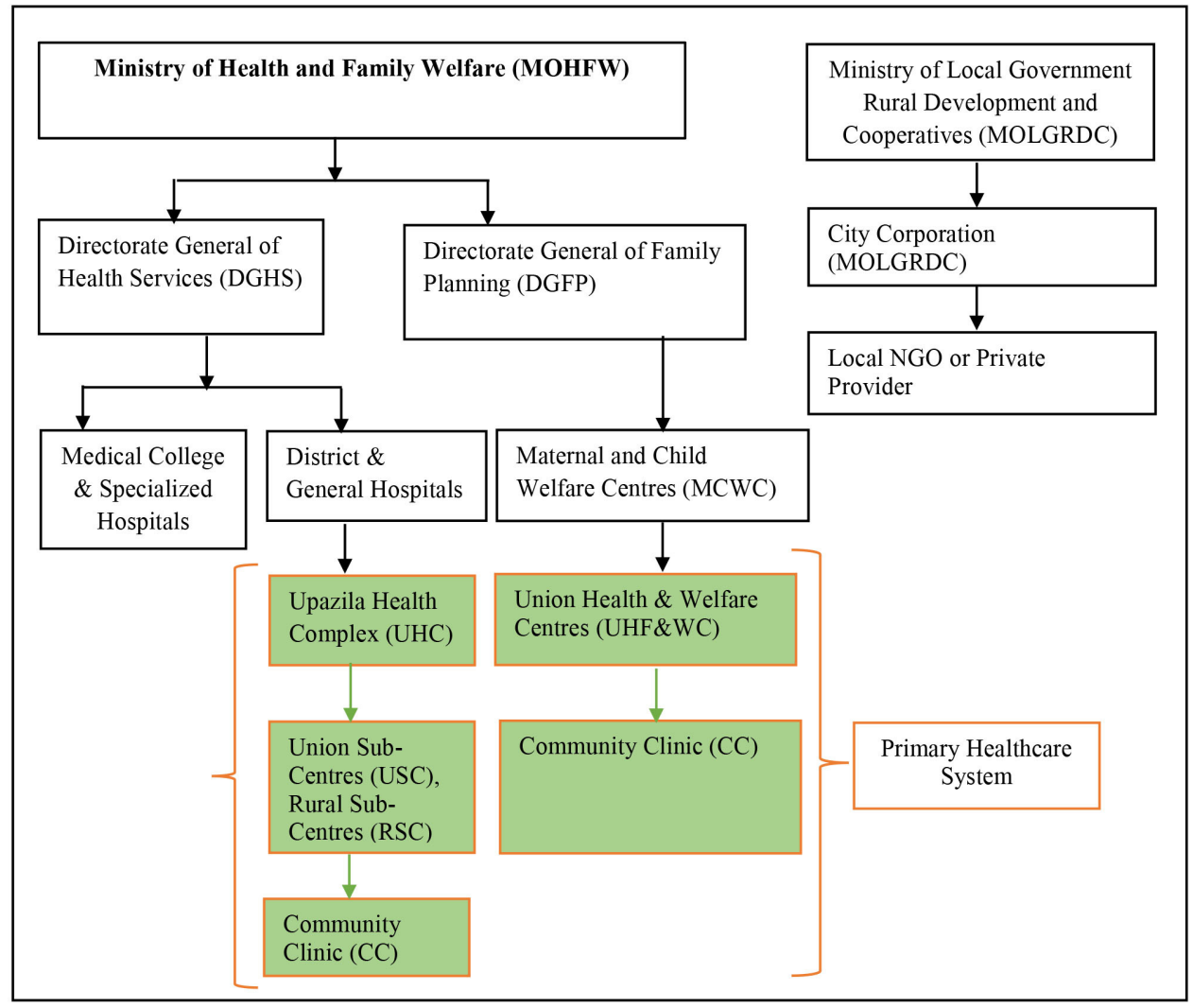

Figure 1 Health service delivery organisational structure in Bangladesh. Adapted from Bangladesh Health System Review. ${ }^{49}$ ngo, non-governmental organisation.

\section{Outcomes measure}

The outcomes of the current study will be the readiness (availability and functions) of a set of supportive items that have been identified in the WHO PEN package, and indicators proposed in the WHO SARA methodology. These items will be measured under three major categories: (1) staff and guidelines, (2) basic medicine and commodities and (3) diagnostic equipment. Additionally, the capacity of several health system components, and community contexts that influence NCDs-related service organisation and delivery will be assessed, as presented in table 1 .

\section{METHODS AND ANALYSIS}

\section{Study context, PHC system and health service delivery in}

\section{Bangladesh}

Bangladesh's health system is pluralistic, wherein multiple actors and providers play roles by applying a mixed system of medical practices. ${ }^{48}$ The Ministry of Health and Family Welfare is the apex body for designing, formulating and overseeing health relation actions, and has two divisions: health services, and medical education and family welfare. ${ }^{49}$ The health services are provided by four key formal providers: the government or public sector, private operators for profit, non-governmental organisations (NGOs) and charities (notfor-profit) or donor agencies (developing partners/aids). Apart from these, there is an extensive pervasiveness across the country of informal healthcare providers (ie, traditional healers, faith healers, herbalists, quacks and homeopaths).
According to its administrative structure, Bangladesh has approximately 87310 villages, 40977 wards, 4553 unions, 490 upazilas, 64 districts, 4 metropolitan cities and 8 divisions). ${ }^{41}$ Based on this, the healthcare services are organised and delivered through three levels: tertiary, secondary and primary levels. ${ }^{49}$ The tertiary-level facilities mostly exist at the divisional and national levels, providing specialised and advanced care. The secondary level exists at the district level, providing specialised care in addition to PHC. Finally, the primary care level exists at the upazila level, which provides basic healthcare services. ${ }^{3449}$ There are various type of healthcare facilities in the primary care level including 'community clinics (CC)' located at the village/ward, Union Health and Family Welfare Centers, Rural Health Clinics (RHC) and union subcentres (USC) are set up at the union level (several villages comprise a union, which is the lowest administrative unit in Bangladesh). The UHC hospitals are situated at the upazila headquarters (several unions comprise a upazila, and a few upazilas comprise a district) (figure 1). According to the latest data, there are 420 UHC hospitals with 18432 inpatient beds (31-50 beds per UHC) across the country, which are linked to the district-level hospitals (100-250 beds) (table 2). ${ }^{50}$

\section{Study population}

Bangladesh's health system is considerably uniform in terms of health service delivery, organisation of the healthcare workforce, logistics and supplies. However, distinctive sociodemographic characteristics, geographical features, 
Table 2 Primary healthcare facilities in the public sector in Bangladesh

\begin{tabular}{|c|c|c|c|}
\hline Level & Type of facility & Type of service & No of facilities (n) \\
\hline \multirow[t]{7}{*}{ Upazila } & Upazila health complex (50 beds) & Hospital & 297 \\
\hline & Upazila health complex (31 beds) & Hospital & 112 \\
\hline & & Total of upazila health complexes & 420 \\
\hline & Hospitals outside health complexes & & \\
\hline & 30-bed hospital & Hospital & 1 \\
\hline & 25-bed hospital & Hospital & 1 \\
\hline & Mother and child welfare centre & Hospital & 12 \\
\hline \multirow[t]{6}{*}{ Union } & Union-level facilities under DGHS & & \\
\hline & 20-bed hospital & Hospital & 32 \\
\hline & Union health and family welfare centre & Outpatient only & 87 \\
\hline & Union-level facilities under DGFP & & \\
\hline & Mother and child welfare centre & Hospital & 24 \\
\hline & Union health and family welfare centre & Outpatient only & 3924 \\
\hline Ward & Community clinic & Outpatient only & 13442 \\
\hline
\end{tabular}

Adapted from Bangladesh Health Facility Survey 2017. ${ }^{73}$

DGFP, Directorate General of Family Planning; DGHS, Directorate General of Health Services.

livelihood patterns and sociocultural practices may affect different health outcomes. Taking this variation into account, this study will cover various regions applying a multistage cluster random sampling approach. At the first step of this process, four administrative divisions (out of eight) were randomly selected: Dhaka, Khulna, Rajshahi and Sylhet. One administrative district was then randomly selected from each division: Cumilla, Jhenaidah Rajshahi and Sylhet, respectively (figure 2). Finally, two subdistricts (locally known as upazila) were selected at random from each of these four districts. Due to various factors such as study budget and field constraints, complex healthcare delivery mechanisms, target groups, resource mobilisation and allocation modality, and relatively small coverage, we decided to exclude metropolitan cities in this study.

\section{Study design and conceptual frameworks}

This study will use a mixed-method study design (figure 3), which will include both quantitative and qualitative approaches. A mixed-method design implies combining quantitative and qualitative approaches to evaluate research questions. ${ }^{51}$ Mixed-method design is viewed as appropriate to get a comprehensive understanding as it examines the observed phenomena from multiple perspectives and to validate findings through triangulation of methods, participants and sources. ${ }^{51}{ }^{52}$ The mixed-method study includes various designs: convergent design, explanatory sequential design and exploratory sequential design. This study will use convergent design by collecting qualitative and quantitative data in parallel, then analysing them separately, and finally merging qualitative and quantitative data. ${ }^{52} 53 \mathrm{~A}$ relevant theoretical framework will be implied to address each objective/ research question.

In the quantitative part, WHO's SARA methodology and PEN Disease Interventions for PHC frameworks will be used to assess the readiness of healthcare facilities to deliver NCD-related services. WHO's SARA methodology offers core indicators of general and service-specific readiness, with service availability (physical presence of services) and readiness (capacity to deliver services) being the two major domains. General and service-specific indicators included (1) trained staff and guidelines, (2) availability of basic equipment, (3) medicines and commodities and diagnostics, and (4) standard precaution for infection control. ${ }^{54}$ Likewise, PEN Disease Interventions for PHC identified a set of core technologies, medicines, risk prediction tools and essential NCD interventions at the PHC in low-resource settings. ${ }^{55}$ These indicators will be used to assess the availability and readiness of relevant health system components (ie, health service delivery, healthcare workforce, supplies and logistics).

In the qualitative component, this study will apply the 'health system dynamics framework' (figure 4) to assess the characteristics of community members and their contexts regarding NCD-related service organisation and delivery. This framework builds on the WHO's previous health system framework, the six building block model and concurrent approaches. ${ }^{56}$ However, the health 


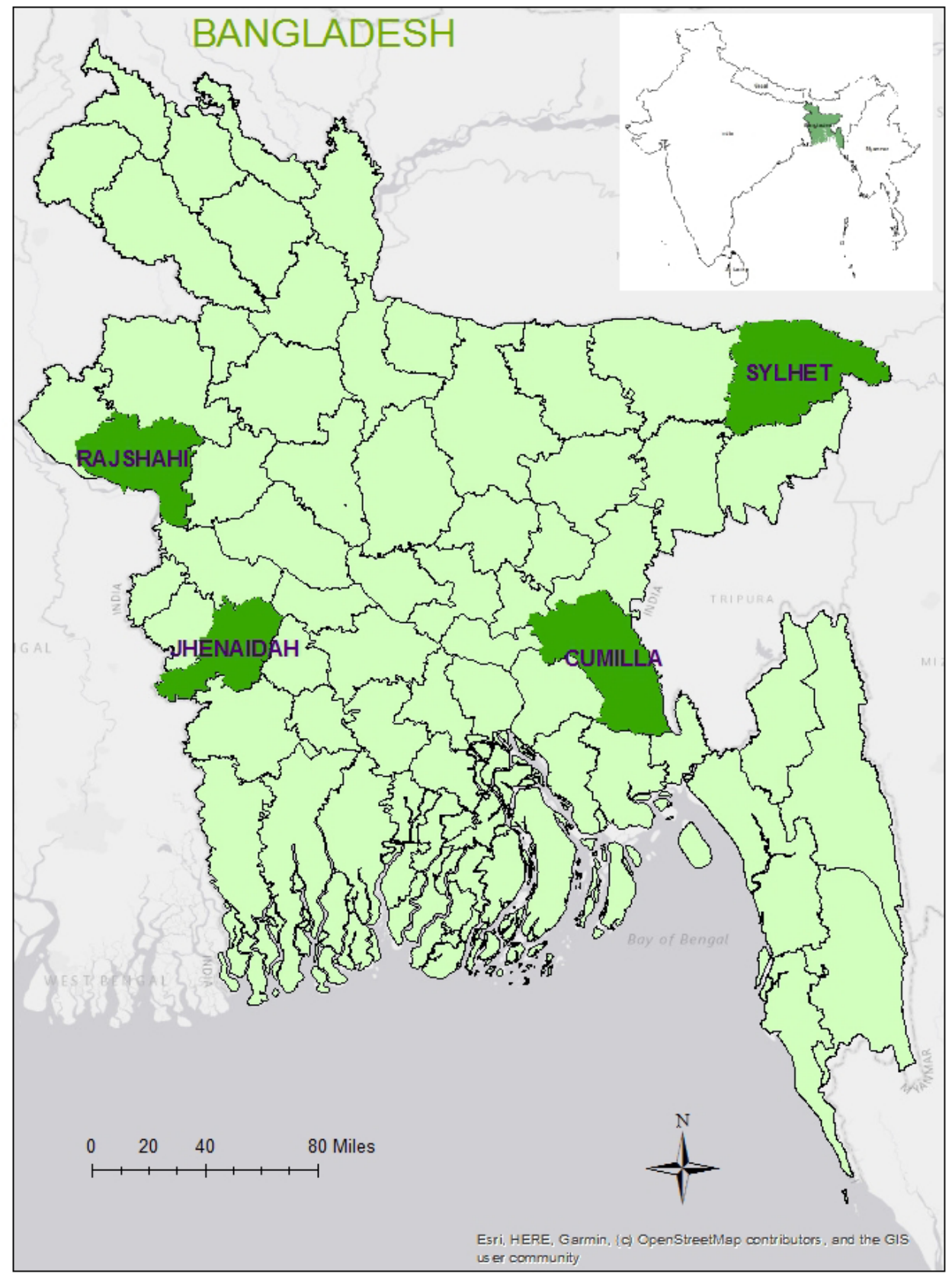

Figure 2 Study site map.

system dynamics framework consists of ten components (ie, goals and outcomes, values and principles, service delivery, population, context, leadership and governance, finance, human resources, infrastructure and supplies, knowledge and information). This framework can adequately explain how and whether different health system elements within a broader societal context interact and influence each other, and how population/individuals' behaviour and choices, and the process affect this mechanism. This framework will offer a comprehensive approach to explore and explain how the PHC system is constructed, and whether it is functioning well within the study context. ${ }^{57}$

\section{Study participants and data collection}

In the quantitative component, we will collect data from primary-level healthcare facilities and households located in the randomly selected eight subdistricts. Using the WHO's SARA and PEN interventions framework, facilitylevel data will be collected from both public (UHC, USC, RHC and CC) and privately-run healthcare facilities (private clinics/hospitals) (table 3) to assess the readiness of the primary-level healthcare facilities to prevent and manage NCDs. The heads of the respective healthcare facilities such as managers, or any designated persons will be interviewed to collect health service data. Additionally, epidemiological, sociodemographic and context-specific data will be collected using household survey methods. 


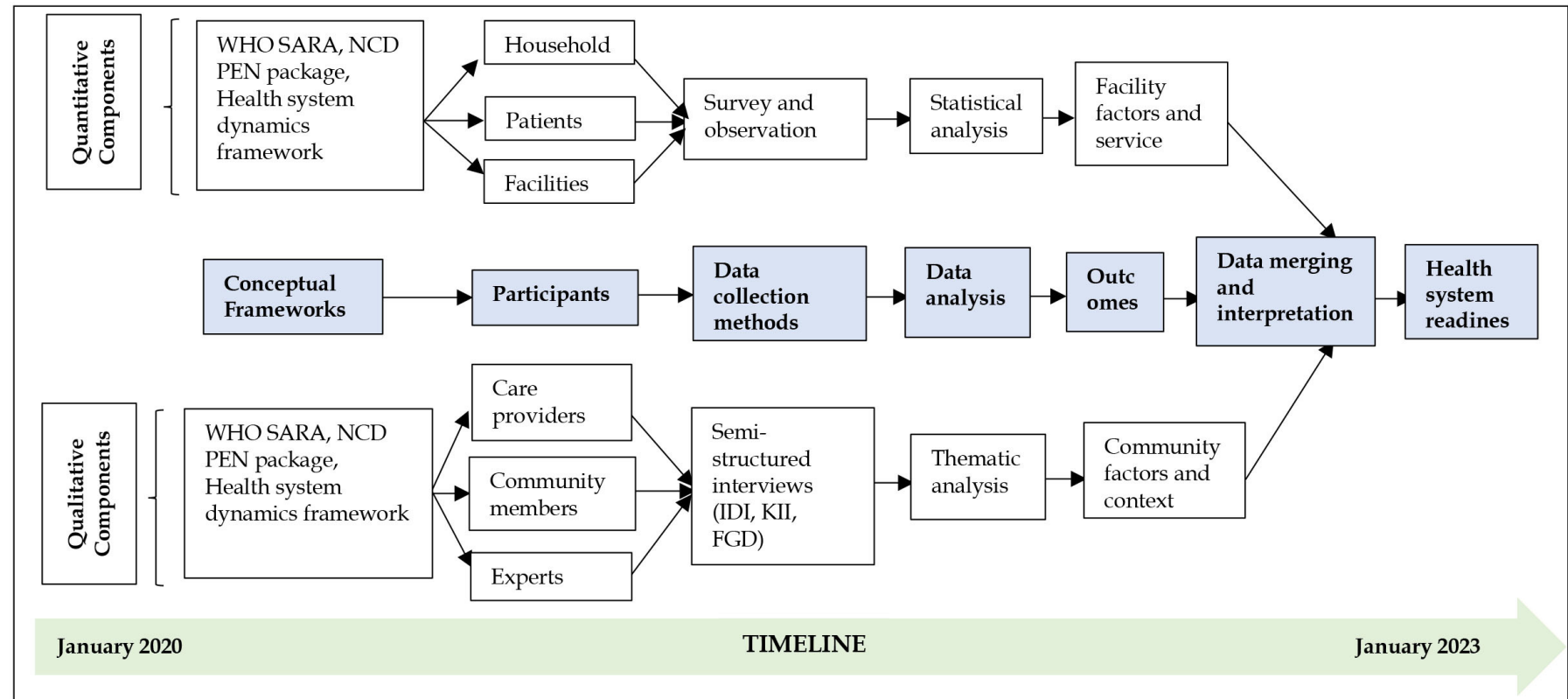

Figure 3 Study design with data collection method and analysis. FGD, focus group discussions; IDI, in-depth interviews; KII, key informant interviews; NCD PEN, non-communicable disease package of essential interventions; SARA, Service Availability and Readiness Assessment.

Adult community members (aged $\geq 18$ years) will be interviewed using cross-section survey design to obtain sociodemographic and NCD-related data from the selected subdistricts.

In the qualitative component, we will collect data from community members, healthcare providers and policymakers regarding the characteristics of communities, and their contexts that influence access to and utilisation of NCD-related services at the primary-level facilities. At the same time, information on the views, perceptions and understanding, life-style factors, perspectives and experiences of communities regarding NCD-related treatment access, adherence, follow-up and referral systems will be collected by interviewing (in-depth interviews (IDIs),

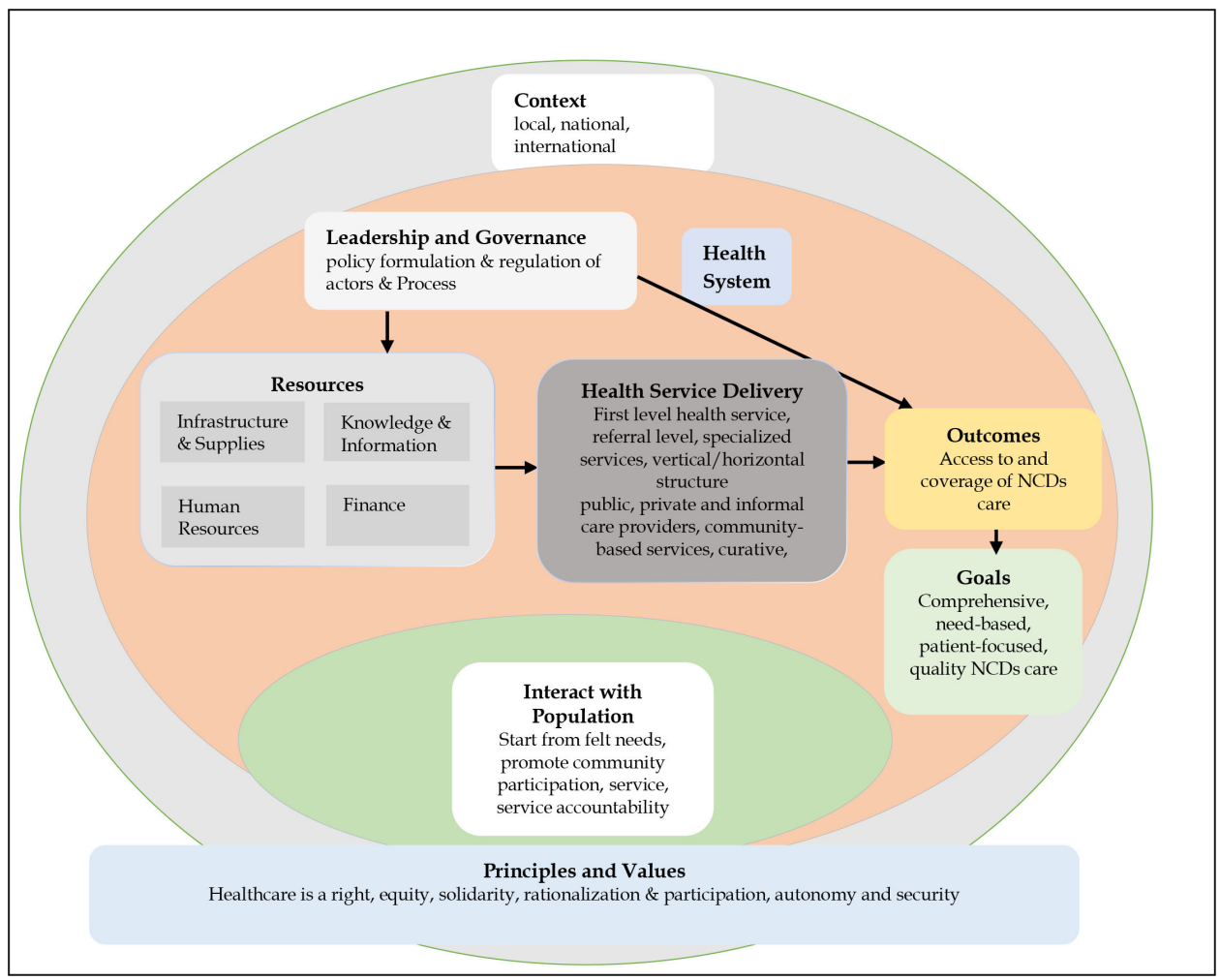

Figure 4 Health system dynamics framework. Source: adapted from van Olmen et al. ${ }^{57} \mathrm{NCDs}$, non-communicable diseases. 
Table 3 Data collection processes and study participants

\begin{tabular}{|c|c|c|c|c|c|c|c|}
\hline \multirow[b]{2}{*}{ Methods } & \multicolumn{6}{|l|}{ Participant(s) } & \multirow[b]{2}{*}{ Other(s) } \\
\hline & Rajshahi & Sylhet & & Cumilla & & Jhenaidah & \\
\hline \multirow{2}{*}{$\begin{array}{l}\text { Household } \\
\text { survey } \\
(n=1386)\end{array}$} & Households $(n=266)$ & Household & $=370)$ & $\begin{array}{l}\text { Households } \\
(n=565)\end{array}$ & & Households $(n=185)$ & $x$ \\
\hline & Tanore & Kanaighat & $\begin{array}{l}\text { Surma } \\
\text { (upazila) }\end{array}$ & Daudkandi & Homna & Harinakunda & \\
\hline $\begin{array}{l}\text { In-depth } \\
\text { interviews } \\
\text { (IDIs)( } n=16)\end{array}$ & \multicolumn{6}{|c|}{$\begin{array}{l}\text { IDI1: with front-line health staff (health assistant/family welfarevisitor/subassistant community medical officer } \\
(n=2) ; \text { IDI2: with private vendor/pharmacist/traditional provider (village doctor, faith healers, kabiraj }(n=2))(\text { In each } \\
\text { upazila, }(n=4)) \text { ) }\end{array}$} & $x$ \\
\hline $\begin{array}{l}\text { Focus group } \\
\text { discussions } \\
\text { (FGDs) }(n=16)\end{array}$ & \multicolumn{6}{|c|}{$\begin{array}{l}\text { FGD1: with community (people with at least one NCD) }(n=2) \text {; FGD2: with community (people with no NCD) }(n=2) \\
\text { (in each upazila, }(n=4) \text { ) }\end{array}$} & $x$ \\
\hline
\end{tabular}

IMS, information management system; NCD, non-communicable disease; NGO, non-governmental organisation.

focus group discussions (FGDs) and key informant interviews (KIIs)) key stakeholders such as policy planners, health administrators, physicians, researchers and epidemiologists. Policy-oriented and programmatic data will be collected through collating and appraising the relevant key policy documents, strategy papers, guidelines, protocols, action plans and related documents.

\section{Sample size calculation}

Quantitative (household survey)

For the household survey, we used the following formula.

Here,

$\mathrm{n}=$ the desired sample size (respondents)

$\mathrm{p}=$ the proportion of the target population. We took the nationally representative data that reported the ageadjusted prevalence of diabetes as $9.7 \%^{58}$ into account, which was the highest in Bangladesh.

$\mathrm{p}=1 \mathrm{p}$

$\mathrm{d}=$ degree of accuracy desired, which is set at (0.02) $2 \%$.

$\mathrm{Z}_{\alpha / 2}=$ the standard normal deviate usually set at 1.96 (corresponds to the 95\% CI)

$$
\begin{gathered}
\mathrm{n}=\frac{\mathrm{Z}^{2} * \mathrm{P}(1-\mathrm{P})}{\mathrm{d}^{2}} \\
\mathrm{n}=\frac{(1.96)^{2} * 0.097(1-0.097)}{(0.02)^{2}} \\
\mathrm{n}=\frac{3.84 * 0.097 * 0.903}{0.0004} \\
\mathrm{n}=\frac{3.84 * 0.087}{0.0004} \\
\mathrm{n}=\frac{0.336}{0.0004}
\end{gathered}
$$

$\mathrm{n}=840$ (respondents).

This calculation provided the minimum required sample size of 840 respondents. Considering the nationwide coverage and sociodemographic heterogeneity of the population, the sample size was multiplied by the design effect of $1.5,{ }^{59}$ to adjust sampling variance caused by the multistage study design,$^{60}$ which resulted in a sample size of $840 \times 1.5=1260$. Additionally, a $10 \%$ non-response rate was applied as it is less than $10 \%$ in individual study, ${ }^{60}$ as well as nationally representative demographic and health surveys. ${ }^{61} 62$ which increased the sample size by 126, resulting in a final sample size of 1386 respondents.

\section{Quantitative (healthcare facility survey)}

We have used the formula (below) taken from the sampling manual for facility surveys developed by MEASURE (Monitoring and Evaluation to ASsess and Use REsults) Evaluation ${ }^{63}$ to calculate sample size for the healthcare facilities.

Here,

$\mathrm{n}=$ the desired sample size $\mathrm{p}=$ anticipated proportion of the facilities with the attribute of interest as $50 \%$, reported by a previous study. ${ }^{64}$

$\mathrm{d}=$ design effect as 1.2 and the width of CI for key estimates at $\mathrm{p} \pm 0.2 \%$.

$\mathrm{V}^{2}=$ the relative variance. It is the square of the relative error taken as $20 \%$.

$\mathrm{Z}_{\alpha / 2}=$ the standard normal deviate usually set at 1.96 (corresponding to the $95 \% \mathrm{CI}$ ) 


$$
\begin{gathered}
\mathrm{n}=\frac{(\mathrm{Z})^{2} * \mathrm{P} * \mathrm{~d}^{2}}{(\mathrm{~V})^{2} * \mathrm{P}} \\
\mathrm{n}=\frac{(1.96)^{2} * 0.05 * 1.2}{(0.02)^{2} * 0.5} \\
\mathrm{n}=\frac{3.84 * 0.6}{0.04 * 0.5} \\
\mathrm{n}=\frac{2.304}{0.02}
\end{gathered}
$$

$\mathrm{n}=115$ (healthcare facilities).

This calculation yielded the minimum required sample size of 115 healthcare facilities. Anticipating a $10 \%$ nonresponse rate, the sample size was further increased by 15 . Thus, the final sample size was 125 .

Sample size for qualitative component (IDIs, FGDs and KIls)

The number of interviews (IDIs, KIIs and FGDs) will be determined on the principle of 'data saturation' (a point of time when no new information, themes, dimensions emerge).$^{65}$ Based on this principle, the final number of interviews may not be determined until the complete list of interviews are conducted. However, we propose to conduct 16 IDIs, 13-15 KIIs and 16 FGDs according to Guest $e$ t $a l \mathrm{~s}^{65}$ proposition to reach data saturation. This number may differ based on the data redundancy noted during the data collection.

\section{Sampling strategy}

Quantitative (household survey)

For household surveys, this study will adopt a multi-stage stratified cluster sampling procedure (figure 5) to ensure nationwide coverage, effective management and supervision of data collection activities. Bangladesh is divided into eight administrative divisions: Barisal, Chittagong, Dhaka, Khulna, Mymensingh, Rajshahi, Rangpur and Sylhet. Each division consists of several districts, and each district is further divided into subdistricts. Each subdistrict has several union parishads in rural areas, which are further subdivided into mouzas. In urban areas, each subdistrict is divided into several mohallas. The entire country has been divided by the Bangladesh Bureau of Statistics (BBS) into 296718 enumeration areas (EAs) according to the EA map in the latest 'Population and Housing Census'. ${ }^{41}$ On an average, each EA consists of 120 households. ${ }^{61}$ For its sampling frame, this study used the list of EAs provided by the BBS. In the first stage, it randomly selected four administrative divisions. From each of the selected divisions, a district was randomly selected. The number of participants required was determined by the proportion of the population in the urban and rural areas. ${ }^{41}$ Based on the latest estimated population

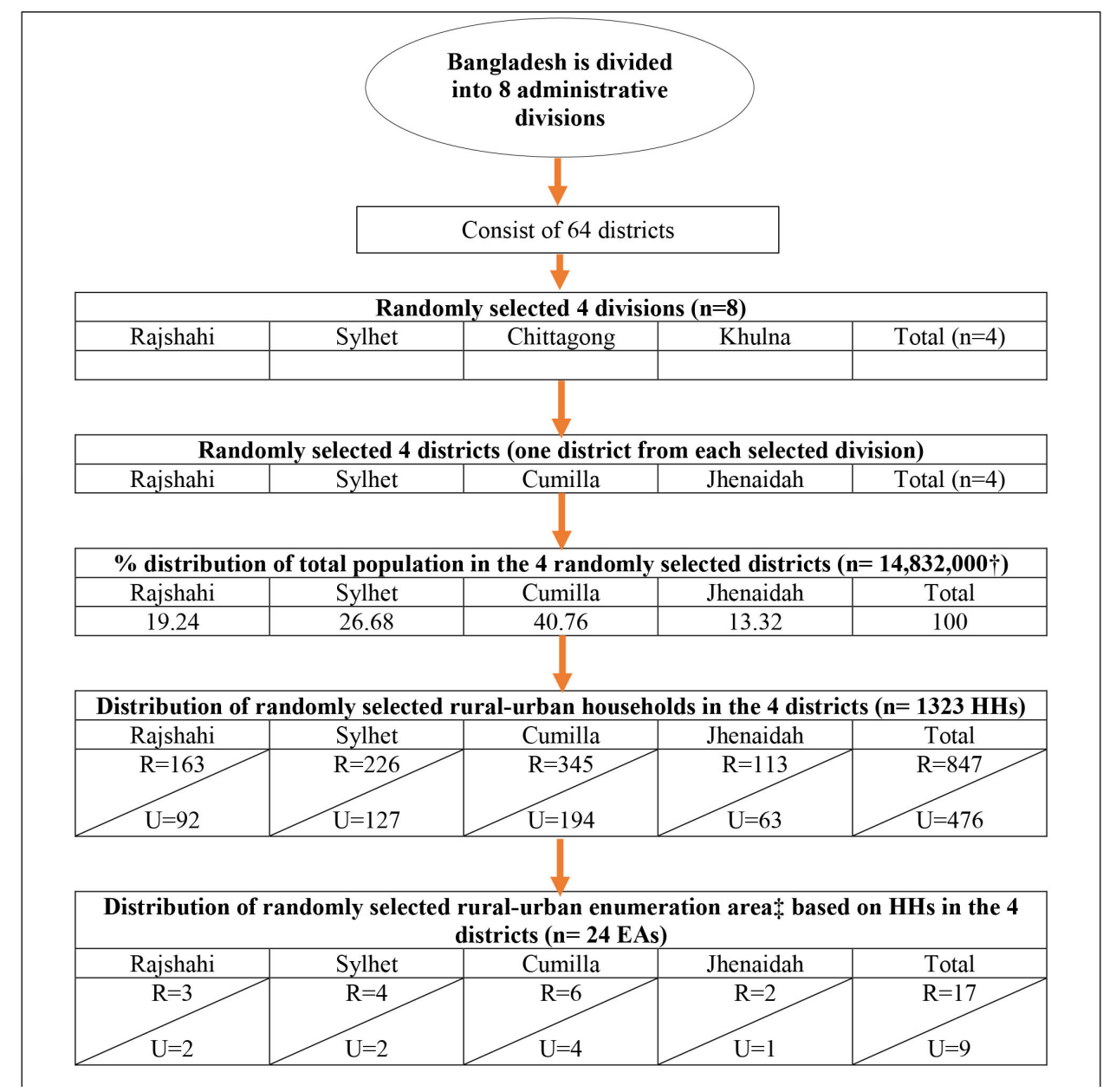

Figure 5 Sampling strategy. EAs, enumeration areas. HHs, household 
in the country, the rural and urban populations were categorised as $36 \%$ and $64 \%$, respectively. ${ }^{66}$ The sample size of 1323 was divided into all the four randomly chosen districts. The smallest number of participants required in any single EA was 63 (in Jhenaidah). This number was considered as the maximum sampling intensity in an available EA. Thereafter, 17 EAs in rural, and 9 EAs in urban areas were randomly chosen. In the next stage, a systematic random sampling procedure will be used to select 63 households, starting from the centre of an EA, which will be located by asking local residents/community members. Field enumerators will reckon the household closest to the centre point as the first household to be enrolled. In the next stage, applying inclusion criteria (age $\geq 18$ years, not pregnant, no history of surgery for the last 3 months) reported by a previous study, an adult aged 18 years and above will be interviewed. ${ }^{28}$ Data will be collected from an eligible member in the selected household following the 'Kish Grid' method, ${ }^{67}$ which will allow us to interview only a single member of the selected household. In case of the unavailability of household members (ie, household shut down during data collection, or decline to participate), the next eligible household will be approached. The second eligible household will be selected by skipping the next household, and choosing the subsequent household (ie, every alternate household). This process will be repeatedly followed until the expected sample size is reached.

\section{Quantitative (healthcare facility survey)}

Healthcare facilities will be randomly selected for facility survey. We will prepare the list of healthcare facilities at the primary care level by collecting basic information (facility type, location, service availability, operating hours, etc) from the 'Directorate General of Health Services' database. From this list, we will randomly select healthcare facilities located at various levels with the variation of size and patient load (eg, UHC, UHC/union subcentre CC, private clinic/hospital, NGO clinic/hospital). Facilitylevel data will be gathered by face-to-face interviewing facility heads/designated personnel.

\section{Qualitative component (IDIs, FGDs and Klls)}

Purposive sampling strategy will be adopted for selecting the interview participants. ${ }^{69}$ Some predefined inclusion criteria ( $(1)$ aged $\geq 18$ years living in the community; (2) persons who are better aware of NCDs (ie, school teachers, faith leaders, village head); (3) person engaged in NCD-related policy formulation or having expertise (policy planners, individual consultants, NGO activities, govt. official); (4) willing to participate voluntarily) will be applied to select the participants. However, we will take the following three basic principles, widely used in purposive sampling, into account ${ }^{22}$ : (1) maximum variation (we will include participant from roles and backgrounds, locations/subdistricts, gender, age), (2) iterative process (we will consider reinterviewing and revisiting the participant if required to probe or validate specific information) and (3) reflexivity (assess the self-roles/researchers).

\section{Data analysis}

Quantitative (household survey)

Household-level quantitative data will be analysed to uncover factors associated with NCD-related service and care seeking. The outcome variable will be the 'care-seeking for NCDs', which will be recorded in the following three categories: no care or self-care, semiqualified professional care, and qualified professional care. The potential explanatory variables will be the individual characteristics (ie, age, sex, education, comorbidity, occupation), household characteristics (ie, socioeconomic status, household size) and contextual characteristics (ie, the distance of the facility from the household, type of residence). The relationship of these variables with 'careseeking for NCDs' will be assessed by employing multiple multinomial logistic regression analyses, as the outcome variable has more than two categories.

\section{Quantitative (healthcare facility survey)}

Facility-level quantitative data will be analysed following the SARA manual to assess service specific readiness of the PHC facilities. ${ }^{54}$ The primary outcome variable is 'readiness' of the PHC system in Bangladesh, where it is measured in binary scale (has two categories: 'ready' and 'not-ready'). The readiness variable will be rated as an index grouped into following three domains as proposed in the WHO SARA methodology: (1) staff and guidelines, (2) diagnostic equipment and (3) medicines and essential commodities. Each of these domains has multiple indicators, which will be measured in nominal scales: 'yes' and 'no'. An index for each domain will be calculated as the mean availability of indicators, which ranges from $0.00 \%$ to $100 \%$. The facility readiness index (composite score) will then be calculated as the mean of all these three domains and a facility will be considered as 'ready' if its index is above $50 \%{ }^{54}$ The binary multiple logistic regression analysis will be performed to evaluate the relationship between the outcome 'readiness' and several potential explanatory variables such as facility type (eg, inpatients, outpatient), facility location (eg, rural, urban) and managing authority (eg, public, private) of the facility.

\section{Qualitative (IDIs, FGDs and KIIs)}

A thematic analysis approach will be used to analyse qualitative data (IDIs, KIIs and FGDs). ${ }^{70}$ Initially, we will transcribe the interviews in verbatim fashion, and subsequently translate them into English. At this step, all authors will independently read the interviews and familiarise themselves with the information and contexts. In the next step, we will code the data in three steps (open code, axial code and selective codes). Once data coding is completed, we will form several clusters (comprise a few codes), and then a few themes (comprise a few clusters). Finally, we will present the findings under several themes. 
A stepwise procedure will be followed in this process. ${ }^{70}$ All authors will independently prepare some codes and themes, and any disagreements will be resolved following a discussion or consensus. Text management software (ie, NVivo, Atlas-ti) will be used to analyse data. A triangulation of methods and participants will be performed to increase data validity and research rigours.

\section{Patient and public involvement}

No patient involved.

\section{Participants' consent}

At the beginning of the interview, data collectors will inform participants about the purpose of the study. An explanatory Statement will then be provided by to the participants, which they can read, and then ask any questions they have. Participants are then required to read and sign a consent form, agreeing to participate. The consent form (written in Bangla) will explain the purpose of the study, the freedom to participate and how participants' information will be used while maintaining individual/ facility confidentiality.

\section{Workshop for data collection team and pilot study}

Four university graduates with a background in research methods as well as data collection tools and techniques will be hired on an ad hoc basis. The first and second authors will train them on data collection methods and techniques, and use the Research Electronic Data Capture (REDCap) for collecting and recording data, and administering the questionnaire. ${ }^{71}$ REDCap is a secure web-based application for completing electronic surveys and data collection for research studies. It provides (1) a user-friendly interface with validated data entry; (2) audit trails for tracking data manipulation and (3) an automated export procedure for seamless data downloads to standard statistical packages. All questionnaires, observation checklists and interview guidelines will be piloted in Sylhet and Rajshahi districts (beyond these sampled eight districts) to assess their comprehensibility, appropriateness and conformability to the participants and interviewers. Based on the piloting, required changes will be made before finalising the questionnaires, observation checklists and interview guidelines.

\section{Quality assurance measures for data collection}

To ensure data quality, various steps such as accuracy, relevance, completeness and consistency of data will be monitored and checked by all investigators via REDCap application. In addition, the student investigator will carry out a random consistency check for at least 5\% of the interviewed questionnaires. The student investigator will observe the interviews and group discussion session by physical participation in the interview sessions. If physical presence of the investigator becomes limited, the interview sessions will be observed via audio-visual technique (Zoom Video Communication, Skype video chat or voice call). Supportive supervision will be provided to the interviewers, if required.

\section{Ethics and dissemination}

The project has been approved by the Monash University Human Research Ethics Committee (Project ID: 27112). The project also received ethical approval from the Bangladesh Medical Research Council (Ref: BMRC/ NREC/2019-2022/270). The ethical standards set by the ethics committee will be fully complied with throughout the research process. Any sensitive and hidden issues will be addressed according to the ethical values and standards established and exercised by the university and/ or institution. The study participants' confidentiality and anonymity will be maintained at all stages by removing their names. During the data collection period, data will be saved in the secure REDCap web-based application hosted at Monash University. The application will be accessible only by the research team. When the data collection is completed, the data will be exported to IBM SPSS statistical package, and will be saved in the secure faculty-allocated network storage (Monash (S:) drive). Participants' identifiers such as names and designations will be removed from the main database and saved in a separate secure electronic folder, and will not be used for data analysis. The research findings will be published in scientific public health journals. The published reports will include group data only, and no individual data or information will be shared. Summary of the research findings will be disseminated to the community through news media.

\section{DISCUSSION}

To the best of our knowledge, this is the first hybrid study that aims to provide a deeper analysis and assessment of the PHC system in Bangladesh in the context of NCDrelated services. Although NCDs lists in Bangladesh include some more diseases or conditions (including arsenicosis, mental health disorders, hearing disabilities, birth defects and road injuries), our study will include the major NCDs (diabetes mellitus, chronic respiratory diseases, cardiovascular disease and cancer) prioritised by WHO. Therefore, we did not require to modify any tool to include these locally defined NCDs.

The existing studies mainly investigated supply-side factors to assess the healthcare system readiness for NCDs (ie, medicine, basic amenities, medical products and technologies) as devised in the WHO SARA methodology and/or WHO PEN interventions. However, the demand-side factors, which is an essential consideration in addressing NCDs, remain largely underexplored. The proposed study will examine healthcare system readiness by adopting a mixed-method approach and applying the relevant health system framework. ${ }^{57}$ In this present study, use of a conceptual framework and combining various methods/tools will offer a deeper and comprehensive understanding of the healthcare system's readiness from both supply-side and demand-side perspectives.

Bangladesh's PHC system is quite unitary (overly centralised) in terms of management structure, 
organisational hierarchy, resource allocation, workforce deployment, health-seeking behaviour and sociocultural characteristics of the population. ${ }^{72}$ The proposed study will fill critical information gaps in the NCD-related service organisation and delivery mechanism. Since this study will reflect the holistic features of the PHC system currently functioning at subdistrict level, it will constitute comprehensive information to assess the current health system's extent of preparedness, and whether better NCD services at the population level need to be provided. Therefore, the study findings will benefit policy planners, programme implementers, healthcare professionals and community members to develop a need-oriented, effective and patient-centred NCD services mechanism at the PHC level in Bangladesh. Overall, the study findings will guide public health decisions for NCD prevention and management efforts at the PHC system in Bangladesh and the similar contexts elsewhere.

\section{Twitter Md Nazmul Karim @Karim}

Contributors AK, MNK and BB conceived and designed the study. AK developed data collection tools, will implement data collection activities and prepare the first draft of the manuscript. MNK and BB revised the manuscript. BB provided overall stewardship. The final manuscript has been read and approved by all authors.

Funding The authors have not declared a specific grant for this research from any funding agency in the public, commercial or not-for-profit sectors.

Map disclaimer The inclusion of any map (including the depiction of any boundaries therein), or of any geographic or locational reference, does not imply the expression of any opinion whatsoever on the part of BMJ concerning the legal status of any country, territory, jurisdiction or area or of its authorities. Any such expression remains solely that of the relevant source and is not endorsed by BMJ. Maps are provided without any warranty of any kind, either express or implied.

Competing interests None declared.

Patient and public involvement Patients and/or the public were not involved in the design, or conduct, or reporting, or dissemination plans of this research.

Patient consent for publication Not required.

Provenance and peer review Not commissioned; externally peer reviewed.

Open access This is an open access article distributed in accordance with the Creative Commons Attribution Non Commercial (CC BY-NC 4.0) license, which permits others to distribute, remix, adapt, build upon this work non-commercially, and license their derivative works on different terms, provided the original work is properly cited, appropriate credit is given, any changes made indicated, and the use is non-commercial. See: http://creativecommons.org/licenses/by-nc/4.0/.

ORCID iD

Ashraful Kabir http://orcid.org/0000-0002-5597-6065

\section{REFERENCES}

1 World Health Organization. Noncommunicable diseases: key facts: WHO, 2018. Available: https://www.who.int/news-room/fact-sheets/ detail/noncommunicable-diseases

2 Marrero SL, Bloom DE, Adashi EY. Noncommunicable diseases: a global health crisis in a new world order. JAMA 2012;307:2037-8.

3 World Health Organization. Global status report on noncommunicable diseases 2010: WHO, 2010. Available: https:// www.who.int/nmh/publications/ncd report2010/en/

4 Habib SH, Saha S. Burden of non-communicable disease: global overview. Diabetes Metab Syndr 2010;4:41-7.

5 Beaglehole R, Epping-Jordan J, Patel V, et al. Improving the prevention and management of chronic disease in low-income and middle-income countries: a priority for primary health care. Lancet 2008;372:940-9.

6 World Health Organization. WHO called to return to the Declaration of Alma-Ata Alma Ata, Kazakhstan: WHO, 1978. Available: https:// www.who.int/social_determinants/tools/multimedia/alma_ata/en
7 McKee M, Haines A, Ebrahim S, et al. Towards a comprehensive global approach to prevention and control of NCDs. Global Health 2014;10:74

8 Juma K, Juma PA, Mohamed SF, et al. First Africa noncommunicable disease research conference 2017: sharing evidence and identifying research priorities. J Glob Health 2019;8:020301.

9 Kengne AP, Mayosi BM. Readiness of the primary care system for non-communicable diseases in sub-Saharan Africa. Lancet Glob Health 2014;2:e247-8.

10 Beaglehole R, Bonita R, Alleyne G, et al. Un high-level meeting on non-communicable diseases: addressing four questions. Lancet 2011;378:449-55.

11 Elias MA, Pati MK, Aivalli P, et al. Preparedness for delivering noncommunicable disease services in primary care: access to medicines for diabetes and hypertension in a district in South India. BMJ Glob Health 2017;2:e000519.

12 Islam MR, Laskar SP, Macer D. A study on service availability and readiness assessment of non-communicable diseases using the who tool for Gazipur district in Bangladesh. Bangladesh J Bioethic 2016;7:1-13.

13 Panda R, Mahapatra S, Persai D. Health system preparedness in noncommunicable diseases: findings from two states Odisha and Kerala in India. J Family Med Prim Care 2018;7:565-70.

14 Ghimire U, Shrestha N, Adhikari B, et al. Health system's readiness to provide cardiovascular, diabetes and chronic respiratory disease related services in Nepal: analysis using 2015 health facility survey. BMC Public Health 2020;20:1163.

15 Duong DB, Minh HV, Ngo LH, et al. Readiness, availability and utilization of rural Vietnamese health facilities for community based primary care of non-communicable diseases: a cross sectional survey of 3 provinces in northern Vietnam. Int $J$ Health Policy Manag 2019;8:150-7.

16 Kaufman ND, Rajataramya B, Tanomsingh S, et al. Nurse preparedness for the non-communicable disease escalation in Thailand: a cross-sectional survey of nurses. Nurs Health Sci 2012;14:32-7.

17 Kien VD, Van Minh H, Giang KB, et al. Views by health professionals on the responsiveness of commune health stations regarding noncommunicable diseases in urban Hanoi, Vietnam: a qualitative study. BMC Health Serv Res 2018;18:392.

18 Katende D, Mutungi G, Baisley K, et al. Readiness of Ugandan health services for the management of outpatients with chronic diseases. Trop Med Int Health 2015;20:1385-95.

19 Musinguzi G, Bastiaens H, Wanyenze RK, et al. Capacity of health facilities to manage hypertension in Mukono and Buikwe districts in Uganda: challenges and recommendations. PLoS One 2015;10:e0142312.

20 Mutale W, Bosomprah S, Shankalala P, et al. Assessing capacity and readiness to manage NCDs in primary care setting: gaps and opportunities based on adapted WHO PEN tool in Zambia. PLoS One 2018;13:e0200994.

21 Chowdhury AMR, Bhuiya A, Chowdhury ME, et al. The Bangladesh paradox: exceptional health achievement despite economic poverty. Lancet 2013;382:1734-45.

22 Kabir A, Datta R, Raza SH, et al. Health shocks, care-seeking behaviour and coping strategies of extreme poor households in Bangladesh's Chittagong Hill tracts. BMC Public Health 2019;19:1008.

23 Kabir A, Maitrot MRL, Ali A, et al. Qualitative exploration of sociocultural determinants of health inequities of Dalit population in Dhaka City, Bangladesh. BMJ Open 2018;8:e022906.

24 El-Saharty S, Ahsan KZ, Koehlmoos TL, et al. Tackling noncommunicable diseases in Bangladesh: now is the time. World Bank Publications, 2013.

25 Ahsan Karar Z, Alam N, Kim Streatfield P. Epidemiological transition in rural Bangladesh, 1986-2006. Glob Health Action 2009;2 doi:10.3402/gha.v2i0.1904

26 El Arifeen S, Hill K, Ahsan KZ, et al. Maternal mortality in Bangladesh: a countdown to 2015 country case study. Lancet 2014;384:1366-74.

27 Fottrell E, Ahmed N, Shaha SK, et al. Distribution of diabetes, hypertension and non-communicable disease risk factors among adults in rural Bangladesh: a cross-sectional survey. BMJ Glob Health 2018;3:e000787.

28 Khalequzzaman M, Chiang C, Choudhury SR, et al. Prevalence of non-communicable disease risk factors among poor shantytown residents in Dhaka, Bangladesh: a community-based cross-sectional survey. BMJ Open 2017;7:e014710.

29 Khanam F, Hossain MB, Mistry SK, et al. Prevalence and risk factors of cardiovascular diseases among Bangladeshi adults: findings from a cross-sectional study. J Epidemiol Glob Health 2019;9:176-84. 
30 Zaman MM, Rahman MM, Rahman MR, et al. Prevalence of risk factors for non-communicable diseases in Bangladesh: results from STEPS survey 2010. Indian J Public Health 2016;60:17-25.

31 World Bank. Cause of death, by non-communicable diseases (\% of total) - Bangladesh. Available: https://data.worldbank.org/indicator/ SH.DTH.NCOM.ZS?end=2015\&locations=BD\&start=2000\&view= chart

32 Bangladesh World Health Organization, 2016. Available: https://www. who.int/nmh/countries/bgd_en.pdf

33 Zaman M, Ullah A, Bhuiyan M. Noncommunicable disease prevention and control situation in a primary health care setting of Bangladesh: design and baseline findings of an intervention. Chronic Dis Int 2016;3:1021.

34 Ahmed SM. Non-communicable diseases in Bangladesh: current scenario and future directions: Bangladesh health Watch Secretariat. James P Grant School of Public Health 2017.

35 Biswas T, Pervin S, Tanim MIA, et al. Bangladesh policy on prevention and control of non-communicable diseases: a policy analysis. BMC Public Health 2017;17:582.

36 Bleich SN, Koehlmoos TLP, Rashid M, et al. Noncommunicable chronic disease in Bangladesh: overview of existing programs and priorities going forward. Health Policy 2011;100:282-9.

37 Rawal LB, Biswas T, Khandker NN, et al. Non-communicable disease (NCD) risk factors and diabetes among adults living in slum areas of Dhaka, Bangladesh. PLoS One 2017;12:e0184967.

38 Alam N, Chowdhury HR, Ahmed A, et al. Distribution of cause of death in rural Bangladesh during 2003-2010: evidence from two rural areas within Matlab health and demographic surveillance site. Glob Health Action 2014;7:25510.

39 Zaman MM, Bhuiyan MR, Karim MN, et al. Clustering of noncommunicable diseases risk factors in Bangladeshi adults: an analysis of STEPS survey 2013. BMC Public Health 2015;15:659.

40 Mahmood SAl, Ali S, Islam R. Shifting from infectious diseases to non-communicable diseases: a double burden of diseases in Bangladesh. J Public Health Epidemiol 2013;5:424-34.

41 Bangladesh Bureau of Statistics. Population and housing census 2011: socio-economic and demographic report Dhaka, Bangladesh, 2014.

42 Biswas T, Islam MS, Linton N, et al. Socio-economic inequality of chronic non-communicable diseases in Bangladesh. PLoS One 2016;11:e0167140.

43 Dhillon PK, Jeemon P, Arora NK, et al. Status of epidemiology in the who south-east Asia region: burden of disease, determinants of health and epidemiological research, workforce and training capacity. Int J Epidemiol 2012;41:847-60.

44 Ministry of Health \& Family Welfare of Bangladesh. Health Bulletin 2019 Dhaka, Bangladesh, 2019. Available: https://dghs.gov.bd/ images/docs/Publicaations/Health\%20Bulletin\%202019\%20Print\% 20Version\%20(2)-Final.pdf [Accessed 12 Nov 2020].

45 Rawal LB, Kanda K, Biswas T, et al. Non-communicable disease (NCD) corners in public sector health facilities in Bangladesh: a qualitative study assessing challenges and opportunities for improving NCD services at the primary healthcare level. BMJ Open 2019;9:e029562.

46 Banik S, Rahman M. Prevalence of overweight and obesity in Bangladesh: a systematic review of the literature. Curr Obes Rep 2018;7:247-53

47 Biswas T, Haider MM, Das Gupta R, et al. Assessing the readiness of health facilities for diabetes and cardiovascular services in Bangladesh: a cross-sectional survey. BMJ Open 2018;8:e022817.

48 Islam A, Biswas T. Health system in Bangladesh: challenges and opportunities. Am J Health Res 2014;2:366-74.

49 World Health Organization. Bangladesh health system review. Manila: WHO Regional Office for the Western Pacific, 2015.

50 Ministry of Health \& Family Welfare of Bangladesh. Health Bulletin 2017. Mohakhali, Dhaka, 2017.
51 Kaur M. Application of mixed method approach in public health research. Indian J Community Med 2016:41:93.

52 Regnault A, Willgoss T, Barbic S, et al. Towards the use of mixed methods inquiry as best practice in health outcomes research. $J$ Patient Rep Outcomes 2017;2:19.

53 Tariq S, Woodman J. Using mixed methods in health research. JRSM Short Rep 2013:4:2042533313479197.

54 World Health Organization. Service availability and readiness assessment (SARA): an annual monitoring system for service delivery: reference manual. World Health Organization, 2013.

55 World Health Organization. Package of essential noncommunicable (PEN) disease interventions for primary health care in low-resource settings, 2010.

56 World Health Organization. Everybody's business-strengthening health systems to improve health outcomes: WHO's framework for action, 2007

57 van Olmen J, Criel B, Bhojani U, et al. The health system dynamics framework: the introduction of an analytical model for health system analysis and its application to two case-studies. Health $\mathrm{Cul} \mathrm{Soc}$ 2012;2:1-21.

58 Akter S, Rahman MM, Abe SK, et al. Prevalence of diabetes and prediabetes and their risk factors among Bangladeshi adults: a nationwide survey. Bull World Health Organ 2014;92:204-13.

59 Henry GT. Practical sampling. Sage, 1990.

60 Islam RM, Bell RJ, Billah B, et al. The prevalence of symptomatic pelvic floor disorders in women in Bangladesh. Climacteric 2016;19:558-64.

61 National Institute of Population Research and Training (NIPORT). Bangladesh demographic and health survey 2014: full report: Ministry of health and family welfare. Available: https://dhsprogram. com/pubs/pdf/FR311/FR311.pdf

62 National Institute of Population Research and Training (NIPORT). Bangladesh demographic and health survey 2017-18. Dhaka, Bangladesh, 2020.

63 Turner AG, Angeles G, Tsui AO, et al. Sampling manual for facility surveys for population, maternal health, child health and STD programs in developing countries, 2000.

64 Shawon MSR, Adhikary G, Ali MW, et al. General service and child immunization-specific readiness assessment of healthcare facilities in two selected divisions in Bangladesh. BMC Health Serv Res 2018;18:39.

65 Guest G, Bunce A, Johnson L. How many interviews are enough? An experiment with data saturation and variability. Field Methods 2006;18:59-82.

66 Desa U. World population prospects 2019: highlights. New York, NY: United Nations Department for Economic and Social Affairs, 2019.

67 Kish L. A procedure for objective respondent selection within the household. J Am Stat Assoc 1949;44:380-7.

68 Kabir A, Maitrot MRL. Factors influencing feeding practices of extreme poor infants and young children in families of working mothers in Dhaka slums: a qualitative study. PLoS One 2017;12:e0172119.

69 Kabir A, Miah S, Islam A. Factors influencing eating behavior and dietary intake among resident students in a public university in Bangladesh: a qualitative study. PLoS One 2018;13:e0198801.

70 Braun V, Clarke V. Using thematic analysis in psychology. Qual Res Psychol 2006;3:77-101.

71 Harris PA, Taylor R, Thielke R, et al. Research electronic data capture (REDCap) - a metadata-driven methodology and workflow process for providing translational research informatics support. J Biomed Inform 2009;42:377-81.

72 World Health Organization. Bangladesh. Available: https://www.who. int/workforcealliance/countries/bgd/en/ [Accessed 23 May 2021]

73 National Institute of Population Research and Training (NIPORT). Bangladesh health facility survey 2017: final report. Dhaka, Bangladesh, 2017. 